\title{
An Incremental-Hybrid-Yager's Entropy Model for Dynamic Portfolio Selection with Fuzzy Variable
}

\author{
Yin Li, ${ }^{1}$ Jian Tao $\mathbb{D}^{1},{ }^{1}$ and Yazhi Song ${ }^{2}$ \\ ${ }^{1}$ Key Laboratory of Applied Statistics of MOE, School of Mathematics and Statistics, Northeast Normal University, \\ Changchun, Jilin 130024, China \\ ${ }^{2}$ Business School, Jiangsu Normal University, Xuzhou, Jiangsu 221116, China
}

Correspondence should be addressed to Jian Tao; taoj@nenu.edu.cn

Received 12 August 2018; Revised 21 October 2018; Accepted 8 November 2018; Published 2 December 2018

Academic Editor: Allan C. Peterson

Copyright (c) 2018 Yin Li et al. This is an open access article distributed under the Creative Commons Attribution License, which permits unrestricted use, distribution, and reproduction in any medium, provided the original work is properly cited.

\begin{abstract}
To settle down the resolutional uncertainty in optimum portfolio strategy, this paper addresses an incremental-hybrid-Yager's entropy model to newly describe the relationship between return and risk. Different from the traditional multiperiod portfolio, we design the ratio threshold to divide asset price into different time interval and use state instead of time point to model the dynamic portfolio process. In addition, fuzzy variables are utilized to represent prices of assets, while historical data based on Markov chain is exploited to estimate membership functions of fuzzy prices. At last, a compromised genetic algorithm is designed, and the numerical example shows that the proposed model achieves solid returns compared against the mean-variance model and Markov chain Monte Carlo method.
\end{abstract}

\section{Introduction}

Since the original work of Markowitz [1], researches on mean-variance (MV) models for portfolio selection have increased so as to provide financial models with more realistic assumptions. The main goal of the MV model is to maximize the expected return for a given level of risk or minimize the expected risk for a given level of return. Based on the prior work of Markowitz, many extensions have been proposed, such as Giove et al. [2], Gupta et al. [3], and Zhang et al. [4]. For these models, investment horizon is a single period. Because of the investors' requirement for timely wealth reallocation, a number of scholars have extended the classical MV model to multiperiod portfolio selection, in which the objective aims to select a set of intermediate portfolios instead of just the one originally proposed [59]. However, the precondition of multiperiod MV model is that the return of each asset follows the jointly elliptically (or spherically) distributed. This assumption appears to be unrealistic because it rules out possible asymmetry in return distribution, and only a small part of portfolio problem can be addressed by using the MV model. To break down the limitation of traditional methods in asset return distribution, this paper constructs a multiperiod discrete portfolio scheme based on entropy models to optimize portfolio strategy with automatic investment ratio adjustment.

The precondition of maximizing portfolio return is to recognize the systematical risk (uncertainty). An economic system regularly consists of three uncertainties: random uncertainty, fuzzy uncertainty, and resolutional uncertainty [10]. Similar to the result of the dice throwing, price trend has characteristic of randomness, which is called Probability Uncertainty (PU). And plenty of prior studies focus on $\mathrm{PU}$, willing to predict the price process by proposing a large number of models [11, 12]. However, these models are always limitedly used because the price probability is always unknown. Instead, the range $[\underline{u}, \bar{u}]$ of the price trend can be easily obtained rather than the probability of each value within this range. Under this circumstance, fuzzy uncertainty (FU) is considered to describe the price trend, helping make efficient policy to control market risk [13, 14]. Specifically, scholars use the fuzzy set to emphasize the role of expert experience in judging market environment. They conclude that the fuzzy set is the core tool for simulating prior knowledge by models, and it performs well during the process of market decision $[15,16]$. Currently, some researchers realized 
that combining PU and FU can better explain price volatility and simulate price process [17]. However, an overlook factor between PU and FU weakens the prediction accuracy of PUFU models, which is called the resolutional uncertainty (RU). To better understand this uncertainty, we guess the result number of a die has a high probability of 1 or 2; namely, the top face is either 1 or 2 with belief of 0.9 . And this nonspecificity situation which specifies the exact solution by ambiguity is called RU [18]. Since the decision process of asset selection has the same characteristic as dice number guessing that the long-term price prediction is hard to guarantee the accuracy, investors always design a reasonable investment strategy by combining both price historical regulation (PU) and investment experience (FU). Accordingly, the RU is divided into two uncertainties which are independent of each other in optimization algorithm.

To maximize the portfolio return originated from investors prior investment knowledge and minimize the adverse impact of RU on investment decision, the contribution of this paper is to add the description of RU uncertainty and propose a new dynamic strategy for portfolio adjustment to better control the market risk. Specifically, we first use the ratio threshold to divide asset price into different time intervals, and reappraise the market risk according to the time point which price surpass the range of threshold value. Second, the price time point which exceeds the threshold is defined as portfolio state. The investment strategy of each state remains constant, while the algorithm readjusts the asset investment proportion based on the historical price information on state-change point. The ratio threshold usage and the state algorithm design expand the adjustment criterion of portfolio strategy from readjust the portfolio strategy only on the basis of the historical return to refind the optimal investment proportion with the combination of historical income and investor experience, which fills the gap of existing portfolio literatures that lack the description of expert experience. In brief, the set of ratio threshold is the result of historical price trend; meanwhile, it also includes evaluation of subjective judgment for future economic circumstance, which fully describes the RU in real market.

When establishing portfolio models, previous work points that entropy, which is a measurement for the chaos of system, can be well used in simulating the uncertainty of capital price shocked by information from different sources (Rodder et al. [19]; Usta and Kantar [20]; Huang [21]; Xu et al. [22]). Using the principle of maximum, Jana et al. [23] and Zhou et al. [24] point that the entropy can derive the PU of security returns for investors who have limited information under different investment circumstances. In addition, scholars further propose different kinds of entropies to describe the systematic risk in economic system, such as the hybrid entropy and fuzzy cross-entropy (Fang et al. [25] and Jana et.al [23]). Since uncertainties are triggered by not only the external shock but also the internal diversity, Yu et al. [26] propose Yager's entropy, which aims to minimize the distance between assets portfolio ratio and equally proportion can make a good performance in hedging unsystematic risk (internal diversity). To maximize the investors' return, in this paper, we use the incremental entropy to describe the value-added speed of asset and the cumulative income of the return. To minimize the market risk, we choose hybrid entropy which consists of PU and FU and Yager's entropy to simulate unsystematic and systematic risk, respectively. Different from the traditional portfolio model that only focuses on reducing the systemic risk, the model proposed in this paper more comprehensively examines the impact of nonsystemic risk on investment strategy, and the description of nonsystemic model helps to further diversify the risk which is brought by the centralization of portfolio strategy. In addition, the incremental entropy, which is used to characterize the market return, better fits the actual return of asset price, because the geometric mean return is a true reflection of the capital appreciation.

The rest of the paper is organized as follows. In Section 2, we briefly introduce the definitions for fuzzy random returns, fuzzy expectations, incremental entropy, hybrid entropy, and Yager's entropy which are used in model construction. In Section 3, after specifically introducing the usage of the threshold and state interval, we formulate the possibilistic return and the systematic and unsystematic risk of dynamic portfolio. A state-change portfolio model is then built to minimize the cumulative portfolio risk and maximize the return over the whole trading period in Section 4. Section 5 gives a compromised genetic algorithm to solve the proposed model. In Section 6, we give the comparison analysis among our proposed model, a MV portfolio selection model, and MCMC method by using two numerical examples to illustrate the idea of our model and the effectiveness of the designed algorithm. Finally, some conclusions are given in Section 7.

\section{Preliminaries}

Let us first review some basic concepts about fuzzy variable and entropy, which we need in the following sections.

2.1. Fuzzy Variable. A fuzzy number $A$ is a fuzzy set on real line $\mathbf{R}$ with a normal, fuzzy convex, and continuous membership function of bounded support. A $\gamma$-cut set of $A$ is defined by $A_{\gamma}=\left\{x \in \mathbf{R} \mid \mu_{A}(x) \geq \gamma\right\}$ if $\gamma>0$ and $A_{\gamma}=\operatorname{cl}\left\{x \in \mathbf{R} \mid \mu_{A}(x)>0\right\}$ (the closure of the support of $A$ ) if $\gamma=0$. If $A$ is a fuzzy number then $A_{\gamma}=\left[A_{\gamma}^{-}, A_{\gamma}^{+}\right]$is a compact subset of $\mathbf{R}$ for all $\gamma \in[0,1]$.

The concept of a fuzzy random variable, as in the following definitions and conclusions, applies to a situation when randomness and fuzziness appear simultaneously.

Definition 1 (Liu and Liu [27]). Let $\xi$ be a fuzzy variable; then, the expected value of $\xi$ is defined as

$$
\mathrm{E}[\xi]=\int_{0}^{+\infty} \operatorname{Cr}\{\xi \geq r\} d r-\int_{-\infty}^{0} \operatorname{Cr}\{\xi \geq r\} \mathrm{d} r
$$

provided that at least one of the two integrals is finite, where $\xi$ is a fuzzy variable with membership function $\mu$. Further, for any set $B$ of $\mathbf{R}$, the credibility of $\xi \in B$ is

$$
C r\{\xi \in B\}=\frac{1}{2}\left(\sup _{x \in B} \mu(x)+1-\sup _{x \in B^{C}} \mu(x)\right) .
$$


By Definition 1, a fuzzy variable $\xi$ can be characterized by the crisp numbers $(a, b, c)$ with $a<b<c$. The membership function of $\xi$ is given by

$$
\mu_{\xi}(x)= \begin{cases}\frac{x-a}{b-a}, & a \leq x<b, \\ 1, & x=b, \\ \frac{c-x}{c-b}, & b<x<c, \\ 0, & \text { otherwise. }\end{cases}
$$

The $\gamma$-level set of $A$ can be computed as

$$
\begin{aligned}
A_{\gamma}=\left[A_{\gamma}^{L}, A_{\gamma}^{R}\right]=[a-(1-\gamma) L, c+(1-\gamma) R], & \\
\gamma & \in[0,1],
\end{aligned}
$$

where $A_{\gamma}^{L}$ and $A_{\gamma}^{R}$ are the left-endpoint and right-endpoint of the close internal $A_{\gamma}$ and $[L, R]$ is tolerance interval.

Definition 2 (Campos and Gonzalez [28]). Let $A$ be a fuzzy number and its $\gamma$-cut set be $A_{\gamma}=\left[A_{\gamma}^{L}, A_{\gamma}^{R}\right]$. A's $\theta$-mean is defined as

$$
Q^{\theta}(A)=\int_{0}^{1}\left[\theta A_{\gamma}^{R}+(1-\theta) A_{\gamma}^{L}\right] \mathrm{d} \gamma,
$$

where $\theta \in[0,1]$ reflects the DM's (decision maker's) subjective optimistic-pessimistic degree. Moreover, $Q^{\theta}(A)$ could be regarded as the crisp value of fuzzy number $A$.

Here, by changing the value of the parameter, the DM's optimistic-pessimistic degree can be expressed. Generally, $\theta=1$ means that DM is wholly optimistic (expecting maximal value), while $\theta=0$ reflects absolute pessimism (minimal value). In this paper, we choose $\theta=0.5$ to reflect neutrality.

2.2. Entropy Models. As discussed in introduction, complicated financial instruments exhibit hybridized uncertainties of both random and fuzzy types. This section introduces incremental entropy to describe an optimal portfolio for a given probability of returns and employs hybrid entropy to measure financial risk caused by both randomness and fuzziness simultaneously. Yager's entropy, which can minimize the distance between the weight of assets and equal weight, is used to describe unsystematic risks.

Definition 3 (Ou [29]). Consider the prices of $m$ securities in a portfolio as an $m$-dimensional vector, with the price of the $h$ th security having $n_{h}$ values $(h=1,2, \ldots, m)$. Thus, there are $W=n_{1} \times n_{2} \times \cdots \times n_{m}$ price vectors. We assume that the $l$ th price vector is $d_{l}=\left(d_{l 1}, x_{l 2}, \cdots, d_{l m}\right), l=1,2, \ldots, W$, the current price vector is $d_{0}=\left(d_{01}, d_{02}, \ldots, d_{0 m}\right)$, the probability of occurrence of $d_{l}$ is $p\left(d_{l}\right)$, and the return from the $h$ th security is $R_{h l}$ when the price vector is $d_{l}$. $\beta_{h}$ is the proportion of investment in the $h$ th security. Then,

$$
H_{1}=\sum_{l=1}^{W} p\left(d_{l}\right) \ln \left[\sum_{h=0}^{m} \beta_{h}\left(1+R_{h l}\right)\right]
$$

is called incremental entropy. The essence value of incremental entropy is geometric mean return. Since the geometric mean return is the real increment of asset return in the stock market, we choose the incremental entropy as the criterion for optimizing portfolio selection.

Definition 4 (Shang and Jiang [30]). Let $D(X)$ be the collectivity of sets on a discrete universe of discourse $X=\left\{x_{l} \mid l=\right.$ $1, \ldots, W\}, A \in D(X)$, denoted as $A=\sum_{l=l}^{W} \mu_{l} / x_{l}, \forall x_{l} \in X$, where $\mu_{l}=\mu\left(x_{l}\right)$ is the membership of $x_{l}$ subjects to $A$, and the probability distribution on the universe of discourse $X$ is $P=\left(p_{1}, p_{2}, \ldots, p_{W}\right)$, where $p_{l}$ is the frequency of $x_{l}$. We define the hybrid set as $\bar{A}(P, M)=\sum_{l=1}^{W} p_{l} \mu_{l} / x_{l}, \forall x_{l} \in X$, denoted by $\bar{A}(P, M)=\bar{A}$. Then, we call

$$
\begin{aligned}
H_{2} & =F(A) \\
& =-\sum_{l=1}^{W}\left\{p_{l} \mu_{l} \ln p_{l} \mu_{l}+p_{l}\left(1-\mu_{l}\right) \ln \left[p_{l}\left(1-\mu_{l}\right)\right]\right\},
\end{aligned}
$$

the hybrid entropy of hybrid set $\bar{A}$. The hybrid entropy, which consists of stochastic uncertainty and fuzzy uncertainty, is an effective tool to describe the systematical risk in real market.

Definition 5 (Yager [31]). Yager's entropy is defined as

$$
H_{3}=\left(\sum_{l=1}^{n}\left|\omega_{l}-\frac{1}{m}\right|^{z}\right)^{1 / Z}, \quad Z \geq 1,
$$

where $Z$ is a constant and $Z \geq 1$, measuring the entropy of a probability distribution $\omega$ as some measure of the distance of its vector from the vector of maximal entropy; we denote the probability distribution $\omega_{l}=1 / \mathrm{m}$ by the vector $[1 / \mathrm{m}]$.

Remark 6. Definition 5 aims to minimize the distance between the weight of the invested asset and $1 / \mathrm{m}$ in terms of the portfolio selection; the budget allocates on securities more equally while Yager's entropy is larger. Therefore, Yager's entropy has the minimum value if $\omega_{l}=1 / \mathrm{m}$. Yager's entropy, which is used to optimize the proportion of portfolio capital allocation, effectively characterizes the uncertainty of probability distribution and provide an effective tool to depict the nonsystematic risk of assets [26, 32].

\section{The Formulation of Dynamic Portfolio Selection Problem}

In this section, we first explain how to use the threshold and state interval to describe the RU. Then we use the specific incremental entropy, hybrid entropy, and Yager's entropy under each state interval to indicate the portfolio return, systematical risk and unsystematical risk.

3.1. The Threshold Set and State Interval. Since the existence of transaction cost, investors did not intend to frequently alter the portfolio scheme unless volatilities of stock price exceed their acceptable range. To describe this process, we assume the acceptable volatility range (or threshold) is $[a, b]$, where $a$ 
is a lower bound of price reduction ratio of any holding assets while $b$ is an upper bound of price growth rate of any holding assets. In addition, because investors maintain the portfolio strategy when each asset is within the threshold, we assume the unchanged period of investment strategy to be a state. The time that each asset's price is out of the threshold is defined to be the state-change point; and portfolio strategy should be recalculated at each state-change point.

Using multistate portfolio instead of multiperiod portfolio, we can reduce risks and increase profits, achieving timely loss cut in the decline environment of market price and boost revenue in the boom environment of market price. In brief, the price return, risk, and portfolio process are defined as the state-based equations.

Consider a dynamic-selection-based portfolio selection problem with $\mathrm{m}$ risky assets. The return rates of assets are donated by fuzzy variables. Assume that an investor joins the market at the beginning of period 0 with initial wealth $G(0)$ and, at each state $i$, would get wealth $G(i)$. The investor intends to allocate his/her wealth among $m$ risky assets for $C$ states. Here, the simplest SDE model is used to describe and simulate the price evolvement process, because the adjustment proportions of portfolio strategies are only controlled by the returns including the current return, the historical return, and the expected return and correlation among asset prices has no effect on the results of optimal investment proportion. In addition, since the Brownian motion is a main part of the SDE model to express the random trend of asset price, we use this model to describe the random uncertainty of the portfolio return.

Here, $S_{h}(i)$ is used to express the price of risky asset $h$ at state $i$, which is as follows:

$$
\mathrm{d} S_{h}(i)=r_{h}(i) S_{h}(i) \mathrm{d} t+\sigma_{h}(i) S_{h}(i) \mathrm{d} B(i),
$$

where $B(i)$ is a Brownian motion, $r_{h}(i)$ is a drift process of risky asset $h$ at state $i$, and $\sigma_{h}(i)$ is a known volatility of risky asset $h$ at state $i$. Assume that investors have wealth $Y(i)$ at state $i$; then, we get

$$
\frac{\mathrm{d} Y(i)}{Y(i)}=\sum_{h=1}^{m} \beta_{h}(i) \frac{\mathrm{d} S_{h}(i)}{S_{h}(i)}
$$

where $\beta_{h}(i)$ is the form investment proportion of risky asset $h$ at state $i, i=1, \cdots, C, h=1 \cdots, m$, as discretization of (10) yields

$$
\frac{Y(i)-Y(i-1)}{Y(i)}=\sum_{h=1}^{m} \beta_{h}(i) \frac{S_{h}(i)-S_{h}(i-1)}{S_{h}(i)} .
$$

Through (11), we see the return on portfolio under state $i$ based on the strategies under state $i-1$ and the returns on assets under state $i$.

To obtain the optimal investment proportion in each time period, we assume that the whole investment process is selffinancing; that is, the investor does not invest the additional capital during portfolio selection. To increase profits with low risk, investors should alter the scales of assets based on market fluctuation, which we defined as states above. To make it easier to follow our expositions, we list all the notations that will be used hereafter:

$\beta_{h}(0)$ the initial investment proportion of risky assets at states 0 ;

$\Delta \widetilde{\boldsymbol{\beta}}(0)$ the here-and-now decision variable, where $\Delta \widetilde{\boldsymbol{\beta}}(0)=\left(\Delta \widetilde{\beta}_{1}(0), \Delta \widetilde{\beta}_{2}(0), \cdots, \Delta \widetilde{\beta}_{m}(0)\right)^{\prime} ;$

$\Delta \widetilde{\boldsymbol{\beta}}(i)$ the dynamic feedback adjustment proportion of the portfolio at state $i$, where $\Delta \widetilde{\boldsymbol{\beta}}(i)=\left(\Delta \widetilde{\beta}_{1}(i), \Delta \widetilde{\beta}_{2}(i)\right.$, $\left.\cdots, \Delta \widetilde{\beta}_{m}(i)\right)^{\prime}$;

$\Delta \overline{\boldsymbol{\beta}}(i)$ the nominal feedback adjustment proportion of the portfolio at state $i$ where $\Delta \overline{\boldsymbol{\beta}}(i)=\left(\Delta \bar{\beta}_{1}(i), \Delta \bar{\beta}_{2}(i)\right.$, $\left.\cdots, \Delta \bar{\beta}_{m}(i)\right)^{\prime}$;

$\Theta(i)$ the market relation matrix at the state $i$;

$R_{h}(i)$ the return of risky asset $h$ at the state $i$;

$C$ the whole set of states and the frequency of statechange during $n$ trading periods;

$u_{h}(i)$ the upper bound constraint of $\beta_{h}(i), h=1,2, \ldots$, $m, i=1,2, \ldots, C$.

Since the state decisions are actually conditional decision rules, accordingly, the key issue in the stochastic programming formulation is the choice of which action should be taken in response to past outcomes.

In our approach, we postulate that control action $\Delta \overline{\boldsymbol{\beta}}(i)$ is an affine, strictly causal function of the returns' innovations [7]. Then, the dynamic feedback adjustment based on the deviation rate in the previous time period can be described in the following proposition.

Proposition 7. The investment proportion of risky assets at state $i$ is

$$
\begin{aligned}
& \beta_{h}(i)=\beta_{h}(0) \\
& +\sum_{k=1}^{i}\left(\sum_{j=1}^{m} \theta_{h j}(k-1) \mathrm{E}\left(\mathbf{R}_{j}(k-1)-\widehat{\mathbf{R}}_{j}(k-1)\right)\right. \\
& \left.+\Delta \bar{\beta}_{h}(k)\right),
\end{aligned}
$$

where $\left(\theta_{h j}(i)\right)_{m \times m}$ is the sensitivity of the control action in row $h$ and column $j$ of $\Theta(i), \mathbf{R}(i)=\left(R_{1}(i), R_{2}(i), \cdots, R_{m}(i)\right)^{\prime}$ is the vector of the return of the portfolio at state $i$, and $\widehat{\mathbf{R}}(i)=\left(\widehat{R}_{1}(i), \widehat{R}_{2}(i), \cdots, \widehat{R}_{m}(i)\right)^{\prime}$ represents the vector of the predetermined return of the portfolio at state $i$.

Proof. See Appendix A.

Using Proposition 7, we get that the direct ratio of dynamic proportion is composed of fuzzy returns and nominal proportion adjustments. Fuzzy returns are portrayed as the Markov chain in the following section to overcome the effectiveness of subjective factors by predicting experts' experience. Thus, the dynamic portfolio selection problem can be translated into optimal proportion problem of nominal adjustments of each state. 
3.2. Possibilistic Return. The best nominal proportion is the proportion that helps investors get more returns with low risks. Assume that an investor joins the market at the beginning of period 0 with initial investment proportion $\beta_{h}(0)$ to get $h$ assets. To obtain long optimization returns, investors should employ an appropriate way to portray ultimate returns. Thus, a more objective and testable criterion for assessing portfolios, incremental entropy, is applied to obtain the optimal investment ratios. As this kind of entropy emphasizes the incremental speed of capital [24], incremental entropy has contributed to achieve rapid-growth returns for investors.

In this paper, incremental entropy is presented as the description of asset income. Because the larger the incremental entropy is, the more the investors obtain, we maximize the return function to guarantee profit maximization of each state. Extending the incremental entropy method to the statechange circumstance for measuring the return in a dynamicfeedback-adjustment-based portfolio selection problem, we have

$$
H_{4}=\sum_{i=1}^{C}\left[t(i) \sum_{l=1}^{W} p\left(d_{l}(i)\right) \ln \sum_{h=1}^{m} \beta_{h}(i)\left(1+R_{h l}(i)\right)\right]
$$

where $\sum_{i=1}^{C} t(i)=T$ represents the whole period of exchange and $t(i)$ is the duration of time at state $i$.

To calculate values of $H_{4}$, three variables $\beta_{h}(i), p\left(d_{l}(i)\right)$, and $R_{h l}(i)$ should be determined firstly. With the change of state, it is easy to know the value of $\beta_{h}(i)$. However, the value of $p\left(d_{l}(i)\right)$, which represents the probability of price combination under state $i$, is difficult to obtain directly. Thus, to obtain the value of $p\left(d_{l}(i)\right)$, we divide the interval into $n$ equal parts. Then the frequency of the hth subinterval is calculated as follows:

$$
p\left(d_{l}(i)\right)=\frac{\sum_{h=1}^{m}\left[\beta_{h}(i) p_{h l}(i) \lambda_{h}\right]}{\sum_{h=1}^{m} \lambda_{h} \beta_{h}(i)},
$$

where $p_{h l}(i)$ represents the frequency of the $h$ th subinterval price falling in the $l$ th subinterval and $\lambda_{h}$ is the correlation coefficient weight of asset $h$ in the portfolio:

$$
\lambda_{h}=\frac{\sum_{k=1}^{m-1} \mu_{k}}{\sum_{h=1}^{m} \sum_{k=1}^{m-1} \mu_{h k}}
$$

where $\mu_{h k}$ represents the correlation coefficient between the risky asset $h$ and risky asset $k$ with $k=h+1$.

Given $p\left(d_{l}(i)\right)$, we can obtain the value of fuzzy number $R_{h l}(i)$. As $R_{h l}(i)$ is a fuzzy number random variable, the membership function of $R_{h l}(i)$ is given by Definition 1 ; that is,

$$
\begin{aligned}
\mu_{R_{h l}(i)} & (x(i)) \\
= & \begin{cases}\frac{x(i)-a_{h l}(i)}{b_{h l}(i)-a_{h l}(i)}, & a_{h l}(i) \leq x(i)<b_{h l}(i), \\
1, & x(i)=b_{h l}(i), \\
\frac{c_{h l}(i)-x(i)}{c_{h l}(i)-b_{h l}(i)}, & b_{h l}(i)<x(i) \leq c_{h l}(i),\end{cases}
\end{aligned}
$$

where the parameters $a_{h l}(i), b_{h l}(i), c_{h l}(i)$ denote the lowest return, the end return, and the highest return. Here, $a_{h l}(i), b_{h l}(i), c_{h l}(i)$ are estimated by historical data.

To estimate the parameters, a Markov chain is established using historical data. We select stock trading data during trading period $T$ and record the lowest return $a_{h l}^{e}(i)$, the end return $b_{h l}^{e}(i)$, and the highest return $c_{h l}^{e}(i)$. The value range of $a_{h l}^{e}(i), b_{h l}^{e}(i)$, and $c_{h l}^{e}(i)$ is divided into $N$ equal parts, so that the $j$ th subinterval's middle, left, and right values can be represented by $\tilde{a}_{h l}^{j}(i), \widetilde{b}_{h l}^{j}(i)$, and $\tilde{c}_{h l}^{j}(i)$, respectively. Construct a times transition matrix $D=\left(d_{j k}\right)_{N \times N}$ based on each return's respective numbers, where $d_{j k}$ is statistic transferred times from $j$ th state interval to $k$ th state interval. Now, the state transition matrix can be established as

$$
p=\left[\begin{array}{cccc}
p_{11} & p_{12} & \cdots & p_{1 N} \\
p_{21} & p_{22} & \cdots & p_{2 N} \\
\vdots & \vdots & \ddots & \cdots \\
p_{N 1} & p_{N 2} & \cdots & p_{N N}
\end{array}\right]
$$

where $p_{j k}=d_{j k} / \sum_{k=1}^{N} d_{j k}$.

Establishing the steady-state linear equations, we have

$$
\begin{gathered}
k_{1}=p_{11} x_{1}+p_{12} x_{2}+\cdots+p_{1 N} x_{N}, \\
k_{2}=p_{21} x_{1}+p_{22} x_{2}+\cdots+p_{2 N} x_{N}, \\
\cdots \\
k_{N}=p_{N 1} x_{1}+p_{N 2} x_{2}+\cdots+p_{N N} x_{N}, \\
\sum_{j=1}^{N} x_{j}=1 .
\end{gathered}
$$

where $x_{j}$ represents the probability that the return rate data falls into the $j$ th subinterval.

Solving the equation above, we can obtain the unique solution

$$
\begin{gathered}
k_{1}=q_{1}, \\
k_{2}=q_{2}, \\
\cdots \\
k_{N}=q_{N} .
\end{gathered}
$$

Thus, the probability of assets' returns in each state 1,2 , $\ldots, N$ can be described as $q_{1}, q_{2}, \ldots, q_{N}$, and

$$
\begin{aligned}
& a_{h l}(i)=q_{1} \tilde{a}_{h l}^{1}(i)+q_{2} \tilde{a}_{h l}^{2}(i)+\cdots+q_{N} \widetilde{a}_{h l}^{N}(i), \\
& b_{h l}(i)=q_{1} \widetilde{b}_{h l}^{1}(i)_{1}+q_{2} \widetilde{b}_{h l}^{2}(i)+\cdots+q_{N} \widetilde{b}_{h l}^{N}(i), \\
& c_{h l}(i)=q_{1} \widetilde{c}_{h l}^{1}(i)+q_{2} \widetilde{c}_{h l}^{2}(i)+\cdots+q_{N} \widetilde{c}_{h l}^{N}(i) .
\end{aligned}
$$

Based on the transition probability matrix, returns $a_{h l}(i)$, $b_{h l}(i)$, and $c_{h l}(i)$ can be solved such that $\mu_{R_{h l}(i)}(x(i))$ can be calculated. 
Contributing to the Markov chain, a more reliable return exists in $\mathrm{H}_{4}$ that is not influenced by subjective factors.

We want to obtain the maximum of the multiple of investment benefits. However, incremental entropy $H_{4}$ is a fuzzy random variable that cannot yield the true value of $\mathrm{H}_{4}$. Accordingly, the following proposition gives the maximization of the expectation of $\mathrm{H}_{4}$.

Proposition 8. The expectation of $H_{1}$ is

$$
\begin{gathered}
\mathrm{E}\left(H_{4}\right)=K_{1}\left(\sum_{h=1}^{m} \frac{c_{h l}(i)-a_{h l}(i)}{2} \beta_{h}(0)\right. \\
+\sum_{h=1}^{m} \frac{c_{h l}(i)-a_{h l}(i)}{2} K_{2}(i) \\
\left.+\sum_{h=1}^{m} \frac{c_{h l}(i)-a_{h l}(i)}{2} K_{3}(i)\right),
\end{gathered}
$$

where $K_{1}=\sum_{i=1}^{C} G(i), K_{2}(i)=\sum_{k=1}^{i} \sum_{j=1}^{m} \pi_{h j}(k-1) \mathrm{E}\left(R_{h}(i)-\right.$ $\left.\widehat{R}_{h}(i)\right)$, and $K_{3}(i)=\sum_{k=1}^{i} \Delta \bar{\beta}_{h}(k)$.

Proof. See Appendix B.

In a realistic portfolio, given the existence of costs, investors may still not obtain their returns even after achieving $\mathrm{H}_{4}$ in Proposition 8. Thus, a V-shaped function of differences between the ith state portfolio $\beta(i)=$ $\left(\beta_{1}(i), \beta_{2}(i), \cdots, \beta_{m}(i)\right)$ and the $(i-1)$ th state portfolio $\beta(i-$ $1)=\left(\beta_{1}(i-1), \beta_{2}(i-1), \cdots, \beta_{m}(i-1)\right)$ is assumed to describe the transaction cost for each asset under each state. Then, the total transaction cost of the portfolio under state $i$ can be expressed as

$$
C(i)=\sum_{h=1}^{m} c_{h}(i)\left|\beta_{h}(i)-\beta_{h}(i-1)\right|,
$$

where $c_{h}(i)$ represents the unit transaction cost for risky asset $h$ at state $i$.

In this dynamic feedback optimization setting, the constraint of self-financing is represented by

$$
\mathrm{E}\left[\sum_{h=1}^{m} \Delta \widetilde{\beta}_{h}(i)\right]=0
$$

That is, for all $i=1,2, \cdots, C$, we have

$$
\begin{aligned}
& \sum_{h=1}^{m}\left(\sum_{j=1}^{m} \theta_{h j}(k-1) \mathrm{E}\left[R_{j}(i-1)-\widehat{R}_{j}(i-1)\right]\right. \\
& \left.\quad+\Delta \bar{\beta}_{h}(i)\right)=0 .
\end{aligned}
$$

The expected transaction costs of the portfolio at state $i$ can be expressed as

$$
C(i)=\sum_{h=1}^{m} c_{h}(i)\left|E\left[\Delta \widetilde{\beta}_{h}(i)\right]\right|=\sum_{h=1}^{m} c_{h}(i)
$$

$$
\begin{aligned}
& \cdot \mid \sum_{j=1}^{m} \theta_{h j}(k-1) \mathrm{E}\left[R_{j}(i-1)-\widehat{R}_{j}(i-1)\right] \\
& +\Delta \bar{\beta}_{h}(i) \mid .
\end{aligned}
$$

Consequently, from Proposition 8 and (21) the expectation of incremental entropy $H_{4}$ under state $i$ can be expressed as

$$
\max \left\{\mathrm{E}\left(H_{4}\right)-\sum_{i=1}^{C} \sum_{h=1}^{m} c_{h}(i)\left|E\left[\Delta \widetilde{\beta}_{h}(i)\right]\right|\right\} .
$$

3.3. Hybrid Entropy for Systematic Risk. Traditionally, variance is usually used to measure portfolio risk. However, the shortcomings of variance, such as the normal distribution of securities' return rates, positive and negative deviations, and large computation efforts (which are usually unfeasible in a real security market), require us to use hybrid entropy to describe systematic risks.

Proposition 9. If the return on security $\bar{A}$ is a discrete fuzzy random variable, then the risk of $\bar{A}$ is

$$
\begin{aligned}
F(\bar{A})= & p\left(R_{h}(i)\right) \mu\left(R_{h}(i)\right) \ln \left[p\left(R_{h}(i)\right) \mu\left(R_{h}(i)\right)\right] \\
+ & p\left(R_{h}(i)\right)\left[1-\mu\left(R_{h}(i)\right)\right] \\
& \cdot \ln \left\{p\left(R_{h}(i)\right)\left[1-\mu\left(R_{h}(i)\right)\right]\right\},
\end{aligned}
$$

where $0<p\left(R_{h}(i)\right) \leq 1, \sum_{i=1}^{C} R_{h}(i)=1$, and $\mu\left(R_{h}(i)\right) \in(0,1)$.

Given Definition 1, the expression for $p_{h l}(i), p\left(R_{h}(i)\right)$ can be written such that the expansion of risk modeling is clear. Further, $F(\bar{A})$ denotes the average information value of the security and a large value of $F(\bar{A})$ implies a greater degree of uncertainty in the obtaining of returns (i.e., the risk is higher). When $F(\bar{A})=0$, the risk is the lowest. Let $\mathbf{F}$ denote the distance between $F(\bar{A})$ and 0 ; it is obvious that a large value of $\mathbf{F}$ indicates a higher risk, and vice versa.

Suppose investors input their capital in $m$ securities, with the weight of the $h$ th security being $\beta_{h}(i)$ in each state. Then, the risk of a portfolio of these securities is

$$
f(\mathbf{F}, \boldsymbol{\beta})=\sum_{i=1}^{C} \sum_{h=1}^{m} \beta_{h}(i) F_{h}(i),
$$

where $F_{h}(i)$ is the investment risk of the $h$ th security under state $i$.

An old English proverb-“One should not put all eggs into one basket" - cautions against risk concentration. The diversification degree of a portfolio too is important for an investor to make decisions. Many researches have investigated this problem, such as Fang et al. [25] and Jana et. [23]. Diversification can also be termed as unsystematic risks that can be dispersed through suitable portfolio proportion adjustments. The next section discusses the characterization of these risks. 
3.4. Yager's Entropy for Unsystematic Risk. Yager's entropy, which aims to minimize the distance between assets' portfolio ratio and equally proportion, makes a good performance in assets' diversity. Recent researches have shown that Yager's entropy yield has higher portfolio value than those with Shannon's entropy and those with the minimax disparity model. This is because the models of Yager's entropy reflect the change in market by reallocating assets in the portfolio more effectively, which enhance the diversity of portfolios.

Proposition 10. Yager's entropy of diversity proportion of portfolio is

$$
\begin{aligned}
& \min Y\left(\beta_{h}(i)\right) \\
& =\min \left[\left(1-\frac{1}{m}\right)^{1 / 2}-\left(\sum_{h=1}^{m} \beta_{h}^{2}-\frac{1}{m}\right)^{1 / 2}\right],
\end{aligned}
$$

where $m$ indicates an equal proportion strategy.

Proof. See Appendix C.

\section{Construction of State-Change Portfolio Model}

Because of the existence of uncertainty in portfolio selection, investors may lose their profits or capital due to market risks. Usually, investment risks are composed of two parts: systematic risk and unsystematic risk. In Section 3, hybrid entropy and Yager's entropy are applied to describe systematic and unsystematic risk. However, under the complex uncertainty environment, it is difficult for investors to select the best restriction of each asset. Accordingly, in this section, a new portfolio selection method is proposed to find the optimal investment strategy according to both asset price changes and market periodic waves.

Assume that investors' objective is to minimize the cumulative portfolio risk and maximize the return over all $T$ periods, which are expressed as $C$ state-change investments. The portfolio return at each state must achieve or exceed the given minimum expected level. Thus, the state-change portfolio selection problem can be formulated as the following programming problem denoted by $\left(P_{1}\right)$ :

$$
\begin{array}{ll}
\max & \mathrm{E}\left(H_{4}\right)-\sum_{i=1}^{C} \sum_{h=1}^{m} c_{h}(i)\left|E\left[\Delta \tilde{\beta}_{h}(i)\right]\right| \\
\min & \sum_{i=1}^{C} \sum_{h=1}^{m} \beta_{h}(i) F_{h}(i) \\
\min & \sum_{i=1}^{C} \sum_{h=1}^{m} Y\left(\beta_{h}(i)\right) \\
\text { s.t. } & Q^{\theta}\left(\mathrm{E} H_{4}\right)-\sum_{h=1}^{m} c_{h}(i)\left|E\left[\Delta \widetilde{\beta}_{h}(i)\right]\right| \geq R(i), \\
& 0 \leq \beta_{h}(i) \leq u_{h}(i),
\end{array}
$$

$$
\begin{aligned}
& \sum_{h=1}^{m} \beta_{h}=1, \quad \beta_{h}(i) \geq 0, \\
& i=1,2, \cdots, C ; h=1,2, \cdots, m,
\end{aligned}
$$

where $\left(P_{1}\right)$ represents the given minimum expected return rate of the portfolio at state $i$. For convenience, we denote the feasible region of problem $\left(P_{1}\right)$ as $\beta \in \mathrm{D}$.

Note that problem $\left(P_{1}\right)$ has two competing objectives: maximizing the expected returns on the portfolio over the whole investment horizon while minimizing the system and unsystematic risks in the portfolio in each state. To solve the proposed two objectives, we combine the two problems and use the constrained method to solve the single-objective programming model:

$$
\begin{aligned}
& \max \omega_{1} {\left[\mathrm{E}\left(H_{4}\right)-\sum_{i=1}^{C} \sum_{h=1}^{m} c_{h}(i)\left|E\left[\Delta \widetilde{\beta}_{h}(i)\right]\right|\right] } \\
&-\omega_{2}\left[\sum_{i=1}^{C} \sum_{h=1}^{m} \beta_{h}(i) F_{h}(i)\right] \\
&-\omega_{3}\left[\sum_{i=1}^{C} \sum_{h=1}^{m} Y\left(\beta_{h}(i)\right)\right], \\
& \text { s.t. } \quad \beta \in \mathrm{D},
\end{aligned}
$$

where $\omega_{1}, \omega_{2}$, and $\omega_{3}$ denote the coefficients of an investor's preference and $\omega_{1}, \omega_{2}, \omega_{3} \in[0,1], \omega_{1}+\omega_{2}+\omega_{3}=1$. On the one hand, different expectations lead to different investment strategies. On the other hand, model $\left(P_{2}\right)$ is a complex nonlinear programming problem, which is difficult to find an optimal solution using traditional method.

\section{Compromised Genetic Algorithm}

The solution set of optimal proportion in this paper might contain a large number of effective solutions. Longtime calculation caused by too complex searching process and the difficulty of choosing the optimal investment portion among enormous solutions place an almost unendurable strain for investors. Accordingly, to solve the problem $\left(P_{2}\right)$, the compromise genetic algorithm (GA) is designed to find compromise solutions to help decision-makers quickly and accurately obtain the optimal portfolio proportion.

GA was originally proposed by simulating a natural evolution research optimal method. To represent the target research behavior of distance functions, a compromise approach-based algorithm is used in this paper. According to a certain distance measure between the anti-ideal and ideal points, approximating solutions rely on investors' preference. Further, the definition of ideal point $z^{*}=\left(z_{1}^{*}, z_{2}^{*}, \ldots, z_{q}^{*}\right)$ is

$$
z_{j}^{*}=\sup \left\{f_{j}(x) \mid x \in X\right\}, \quad j=1,2, \ldots, q,
$$

where $X$ is the feasible region in the multiobjective programming problem. 
Given the difficulty in approaching $z^{*}$, we define antiideal points $z \in Z$ to utilize distance function $\operatorname{dis}(z)$ to approximate the optimal solutions

$$
\operatorname{dis}(z, p)=\left\|z-z^{*}\right\|_{p}=\left[\sum_{j=1}^{q}\left|z_{j}-z_{j}^{*}\right|^{p}\right]^{1 / p}, \quad p \geq 1,
$$

where $Z=(R, V, Y)$ denotes the optimal value of $\left(P_{2}\right)$ and $Z^{*}=\left(R^{*}, V^{*}, Y^{*}\right)$ represents the anti-ideal point. Then, for each feasible solution $\beta \in \beta^{p}$, regret function $r(\beta, p, \omega)(p \geq$ 1 ) is defined by the weighted $L_{p}$-norm

$$
\begin{aligned}
& r(\beta, p, \omega)=\left\|Z(\beta)-Z^{*}\right\|_{\omega}^{p} \\
& =\omega_{1}^{p}\left|\frac{\mathrm{E}\left(H_{4}\right)-\sum_{i=1}^{C} \sum_{h=1}^{m} c_{h}(i)\left|E\left[\Delta \widetilde{\beta}_{h}(i)\right]\right|^{p}}{R^{*}-R}\right|^{p} \\
& +\omega_{2}^{p}\left|\frac{\sum_{i=1}^{C} \sum_{h=1}^{m} \beta_{h}(i) F_{h}(i)}{V^{*}-V}\right|^{p} \\
& +\omega_{3}^{p}\left|\frac{\sum_{i=1}^{C} \sum_{h=1}^{m} Y\left(\beta_{h}(i)\right)}{Y^{*}-Y}\right|^{p}
\end{aligned}
$$

where $p \geq 1, \omega_{1}, \omega_{2}, \omega_{3}>0$, and $\omega_{1}+\omega_{2}+\omega_{3}=1$. The weights of $\omega_{1}, \omega_{2}$, and $\omega_{3}$ are provided by decision-makers who use their experience to assign different degrees of importance to the two objective functions in $\left(P_{1}\right)$.

Thus, $\left(P_{2}\right)$ is converted into a single-objective programming problem $\left(P_{3}\right)$ :

$$
\begin{array}{ll}
\min & r(\beta, p, \omega), \\
\text { s.t. } & \beta \in \mathrm{D} .
\end{array}
$$

Therefore, we have replaced the difficult linear/nonlinear mixed integer programming models $\left(P_{3}\right)$ with a model based on proxy ideal points that is easier to evaluate and that selects the best puts for investors.

5.1. Representation and Initialization. The way in which a solution is encoded into a chromosome is a key issue in using GAs. Although solutions to the proposed model are fuzzy numbers, this study opts to use the true number by establishing a Markov chain in a real market to obtain a more proximate result. Given the scope of interval $(a, b]$ based on historical data, we can get the state-change time point in $n$ trading periods. To achieve optimal solution in the whole investment time horizon, each state proportion selection should be optimum. Thus, we first deal with the constraint to the initialization of the representation structure.

Problem $\left(P_{1}\right)$ is a multiobjective programming problem pertaining to feasible region $\beta \in \mathrm{D}$. The match between the solution and the chromosome is through $\beta_{h^{\prime}}(i)=$ $\beta_{h}(i) / \sum_{h=1}^{m} \beta_{h}(i)$ to ensure that the random points satisfy constraint $\sum_{h=1}^{m} \beta_{h}(i)=1$. If it satisfies the constraints of problem $\left(P_{1}\right)$, we accept it as a chromosome. Otherwise, we repeat the above processes until we get a feasible chromosome. Thus, a chromosome vector $\mathbf{K}=\left(\beta_{1}(i), \beta_{2}(i), \cdots, \beta_{m}(i)\right)$ satisfying the constraint is randomly chosen to describe a solution $\boldsymbol{\beta}(i)=\left(\beta_{1}(i), \beta_{2}(i), \cdots, \beta_{m}(i)\right)$ to optimize the problem in state $i$. Further, genes $\left(\beta_{1}(i), \beta_{2}(i), \cdots, \beta_{m}(i)\right)$ are restricted in the interval $[0,1]$. Thus, randomly generating a point from hypercube $[0,1]^{m}$ and testing its feasibility, we set an integer pop_size as the number of chromosomes. Repeat this operation pop_size times; then pop_size chromosomes, $\mathbf{K}_{1}, \mathbf{K}_{2}, \cdots, \mathbf{K}_{\text {pop_size }}$, can be obtained.

5.2. Fitness Function. A fitness function is a particular type of objective function that is used to summarize. As a single figure of merit, this function is to evaluate how close a given design solution in achieving the set aims. It is known that the chromosome with a higher fitness will have more chances of generate an offspring. Specifically, the regret value of $\left(P_{1}\right)$ is given by (32). The fitness function to calculate the fitness of a chromosome is

$$
\begin{aligned}
& \operatorname{eval}\left(\beta_{h}(i)\right) \\
& \qquad=\frac{r^{\max }\left(\beta_{h}(i), p, \omega\right)-r\left(\beta_{h}(i), p, \omega\right)+\varepsilon}{r^{\max }\left(\beta_{h}(i), p, \omega\right)-r^{\min }\left(\beta_{h}(i), p, \omega\right)+\varepsilon},
\end{aligned}
$$

where $\varepsilon$ is a random number $\varepsilon \in(0,1), r^{\max }\left(\beta_{h}(i), p, \omega\right)$, and $r^{\min }\left(\beta_{h}(i), p, \omega\right)$ are the maximum and minimum regret values in the current generation, respectively. Then, the fitness function with $L_{2}$-norm can be expressed as

$$
\begin{aligned}
& r\left(\beta_{h}(i), p, \omega\right) \\
& =\min \sqrt{\omega_{1}^{2} \times\left|\mathrm{E} H_{1}-R^{*}\right|^{2}+\omega_{2}^{2} \times\left|V-V^{*}\right|^{2}+\omega_{3}^{2} \times\left|Y-Y^{*}\right|^{2}} .
\end{aligned}
$$

5.3. Evaluation Function. To prevent the populations from degenerating during iteration, both parents and their immediate offspring are candidates for the new generation. We compare the parents with their offspring by evaluating their fitness values to select the fitter ones to store in a mating pool. After the comparison operation, we can obtain pop_size chromosomes with higher fitness values. Then, we perform the proportional selection operation. The chromosome $n$ is selected for a new population by the following probability $P\left(\beta_{h}^{n}(i)\right)$ :

$$
\begin{aligned}
P\left(\beta_{h}^{n}(i)\right)=\frac{\operatorname{eval}\left(\beta_{h}^{n}(i)\right)}{\sum_{n=1}^{\text {pop_size }} \operatorname{eval}\left(\beta_{h}^{n}(i)\right)} & \\
& \\
n & =1,2, \cdots, \text { pop_size. }
\end{aligned}
$$

In this process, the chromosomes of the current population with higher fitness values have higher chance as the parents to reproduce the offspring.

5.4. Crossover Operation. The crossover process is based on arithmetic crossover. We first denote the crossover probability of the genetic algorithm as $P_{k}$. The crossover operation performs as follows. Generate a random number from interval $(0,1)$ and the chromosome $\mathbf{K}_{n}(n=1,2, \cdots$,pop_size $)$ is selected as a parent provided that $v<P_{k}$. Repeat this 
process pop_size times and $P_{k} \cdot$ pop_size chromosomes are expected to be selected to perform the crossover operation. The crossover operation on $K_{1}$ and $K_{2}$ will generate two offspring $\mathbf{K}_{1}^{\prime}$ and $\mathbf{K}_{2}^{\prime}$ as follows:

$$
\begin{aligned}
& \mathbf{K}_{1}^{\prime}=v \mathbf{K}_{1}+(1-v) \mathbf{K}_{2}, \\
& \mathbf{K}_{2}^{\prime}=(1-v) \mathbf{K}_{1}+v \mathbf{K}_{2} .
\end{aligned}
$$

The procedure of the designed genetic algorithm can be summarized as follows.

Step 1. Input parameters pop_size and $P_{k}$.

Step 2. Initialize the randomly generated pop_size chromosomes.

Step 3. Calculate the evaluation function values for all chromosomes.

Step 4. Perform the selection operation by proportional selection.

Step 5. Update the chromosomes by crossover operations.

Step 6. Repeat the third to fifth steps for given number of cycles.

Step 7. Report the best chromosome as the optimal solution.

\section{Numerical Example}

A numerical example is given to express the idea of our model and the effectiveness of the designed algorithm.

Assume that an investor chooses 8 stocks from the Shanghai Stock Exchange for investment. Shown from Figures 1 and 2 , the average volatility and return trend of the 8 stocks are similar to the average volatility and return trend in the same period of Shanghai Stock Exchange. Accordingly, we can conclude that the selected stocks have generality and representability to study the portfolio optimization model proposed in this paper. Based on historical five-minutes data from January 1, 2018, to April 30, 2018, state changes can be seen with a given value of $(a, b]$. Future securities' return rates are triangular fuzzy random variances, where $R_{h l}(i)=$ $\left(a_{h l}(i), b_{h l}(i), c_{h l}(i)\right)$, and can be calculated by Markov chain mentioned in Section 3. In a real market, the values of stocks and assets change frequently and, as such, increasing and decreasing equity holdings blindly may be a huge risk. As such, longtime statistical data are used to anticipate the fuzzy return. When the return rates in state $i$ fluctuate out of interval $[a, b]$, the fuzzy returns on each asset will be adjusted according to 40 historical data before state $i$. Following the above calculation steps, there are 33 state changes under the selection of interval value $[-0.8 \%, 0.8 \%]$. Table 1 shows the first 10 state-change values and anticipates the return on each asset.

Suppose that an investor's initial wealth is $\mathrm{Y}(0)=10,000$ Yuan and the transaction cost rates of stocks at each state are $c_{h}(i)=0.002(i=1,2, \ldots, 10 ; h=1,2, \ldots, 8)$, and the anticipated returns at each time on each asset are constantly

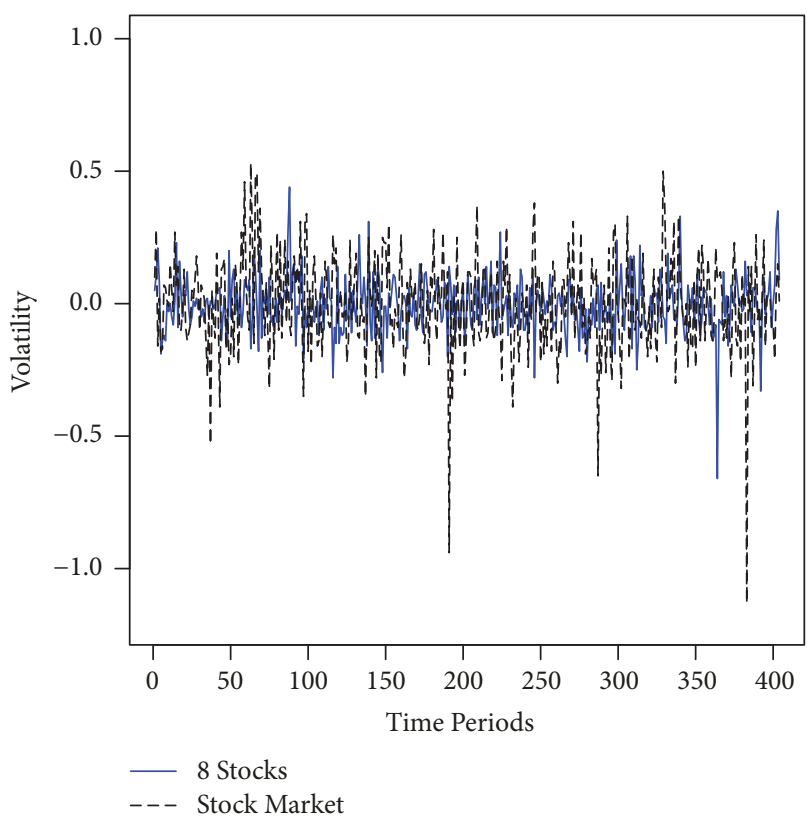

FIGURE 1: Volatility from the entire stock market and from the 8 selected stocks for 400 time periods.

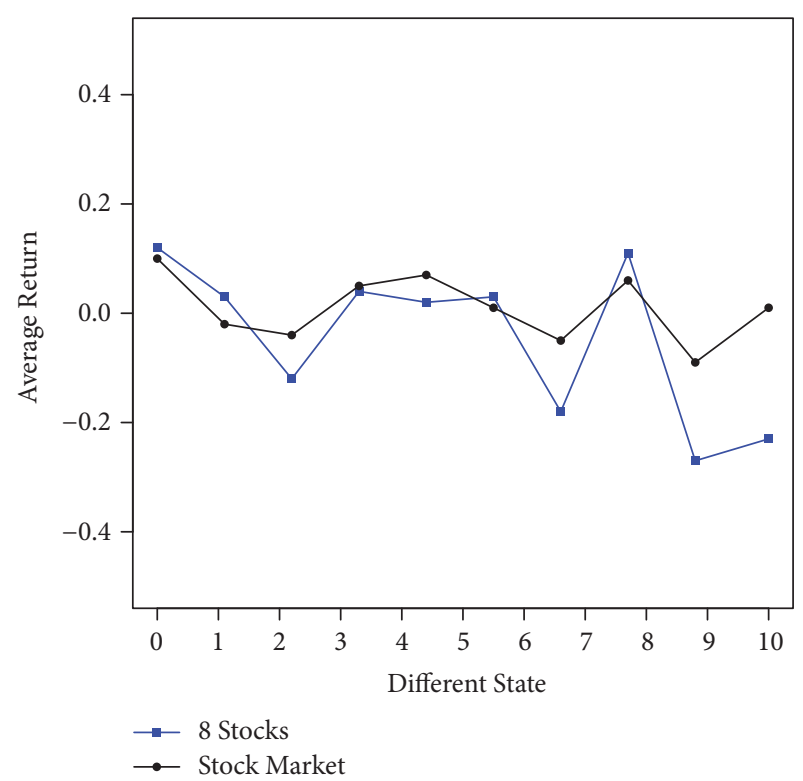

FIgURE 2: Average returns from the entire stock market and from the 8 selected stocks under different states.

$0.05 \%$. Further, we consider that a GA is convergent when the mean regret value is below $0.5 \%$. To show the advantages of the IHYE model, the MCMC method, wherein fuzzy average returns are considered to be a priori estimates, and the classical MV model are used for comparison. Then, based on the values of Table 1 and a priori estimates, the best proportion in each state as given by three methods is shown in Table 2.

Under the compromise genetic algorithm, the average return convergence speed of 8 stocks shown in Figure 3 is fast, and the proportion of the portfolio tends to be stable 
TABLE 1: The expectation rate and the right/left widths of 8 stocks under different states (\%).

\begin{tabular}{|c|c|c|c|c|c|c|c|c|c|}
\hline \multicolumn{2}{|c|}{ No. } & Stock 1 & Stock 2 & Stock 3 & Stock 4 & Stock 5 & Stock 6 & Stock 7 & Stock 8 \\
\hline \multirow{3}{*}{ State 1} & $\mathrm{E}\left(a^{h}\right)$ & 0.33 & 0.91 & 0.19 & 0.42 & 0.2 & 0.32 & 1.66 & 2.14 \\
\hline & $\alpha^{h}$ & 0.12 & 0.19 & 0.10 & 0.06 & 0.35 & 0.01 & 0.26 & 0.14 \\
\hline & $\gamma^{h}$ & 0.19 & 0.27 & 0.17 & 0.12 & 0.09 & 0.28 & 0.24 & 0.26 \\
\hline \multirow{3}{*}{ State 2} & $\mathrm{E}\left(a^{h}\right)$ & 0.89 & 0.38 & -0.011 & 0.01 & 0.10 & 0.40 & 0.17 & 1.80 \\
\hline & $\alpha^{h}$ & 0.17 & 0.05 & 0.14 & 0.09 & 0.06 & -0.01 & 0.14 & 0.32 \\
\hline & $\gamma^{h}$ & 0.21 & 0.14 & 0.23 & 0.11 & 0.18 & 0.77 & 0.17 & 0.27 \\
\hline \multirow{3}{*}{ State 3} & $\mathrm{E}\left(a^{h}\right)$ & 3.12 & 2.58 & 2.15 & -0.89 & 0.24 & -0.20 & 1.05 & 0.58 \\
\hline & $\alpha^{h}$ & 0.19 & 0.09 & 0.14 & 0.06 & 0.02 & 0.17 & 0.19 & 0.42 \\
\hline & $\gamma^{h}$ & 0.61 & 0.51 & 0.26 & 0.12 & 0.55 & 0.23 & 0.19 & 0.81 \\
\hline \multirow{3}{*}{ State 4} & $\mathrm{E}\left(a^{h}\right)$ & 3.32 & 0.98 & 1.17 & 0.82 & 0.21 & 2.44 & 1.18 & -0.30 \\
\hline & $\alpha^{h}$ & 0.29 & 0.25 & 0.14 & 0.06 & 0.14 & 0.08 & 0.24 & 0.77 \\
\hline & $\gamma^{h}$ & 0.61 & 0.35 & 0.23 & 0.12 & 0.49 & 0.33 & 0.60 & 0.95 \\
\hline \multirow{3}{*}{ State 5} & $\mathrm{E}\left(a^{h}\right)$ & 3.61 & 2.41 & 0.60 & 0.67 & 0.35 & 0.03 & 0.45 & 1.10 \\
\hline & $\alpha^{h}$ & 0.10 & 0.09 & 0.21 & 0.08 & 0.06 & 0.22 & 0.38 & 0.21 \\
\hline & $\gamma^{h}$ & 0.30 & 0.51 & 0.27 & 0.19 & 0.76 & 0.56 & 0.61 & 0.45 \\
\hline \multirow{3}{*}{ State 6} & $\mathrm{E}\left(a^{h}\right)$ & 2.87 & 1.89 & 0.57 & 0.42 & 1.10 & 0.23 & -0.20 & 1.70 \\
\hline & $\alpha^{h}$ & 0.21 & 0.17 & 0.21 & 0.09 & 0.32 & 0.19 & 0.07 & 0.32 \\
\hline & $\gamma^{h}$ & 0.31 & 0.53 & 0.32 & 0.17 & 0.68 & 0.33 & 0.30 & 0.27 \\
\hline \multirow{3}{*}{ State 7} & $\mathrm{E}\left(a^{h}\right)$ & 1.71 & 1.29 & 0.14 & 0.41 & 0.89 & 3.19 & 2.21 & -0.47 \\
\hline & $\alpha^{h}$ & 0.09 & 0.19 & 0.02 & 0.09 & 0.21 & 0.07 & 0.27 & 0.25 \\
\hline & $\gamma^{h}$ & 0.21 & 0.71 & 0.09 & 0.19 & 0.42 & 0.56 & 0.68 & 0.44 \\
\hline \multirow{3}{*}{ State 8} & $\mathrm{E}\left(a^{h}\right)$ & 0.28 & 1.30 & 0.47 & -0.35 & -0.03 & 0.17 & 0.22 & 0.91 \\
\hline & $\alpha^{h}$ & 0.04 & 0.18 & 0.15 & 0.11 & 0.27 & 0.19 & 0.29 & 0.21 \\
\hline & $\gamma^{h}$ & 0.18 & 0.67 & 0.15 & 0.17 & 0.52 & 0.22 & 0.47 & 0.99 \\
\hline \multirow{3}{*}{ State 9} & $\mathrm{E}\left(a^{h}\right)$ & 0.71 & 1.40 & 0.31 & -0.47 & -0.02 & 1.21 & 1.45 & 0.20 \\
\hline & $\alpha^{h}$ & 0.11 & 0.11 & 0.10 & 0.21 & 0.29 & 0.14 & 0.49 & 0.11 \\
\hline & $\gamma^{h}$ & 0.19 & 0.39 & 0.17 & 0.25 & 0.34 & 0.44 & 0.32 & 0.35 \\
\hline \multirow{3}{*}{ State 10} & $\mathrm{E}\left(a_{h}\right)$ & 1.49 & 1.29 & 0.28 & -0.68 & -0.01 & 2.11 & 0.90 & 0.30 \\
\hline & $\alpha^{h}$ & 0.08 & 0.10 & 0.11 & 0.16 & 0.21 & 0.11 & 0.66 & 0.27 \\
\hline & $\gamma^{h}$ & 0.22 & 0.39 & 0.24 & 0.21 & 0.75 & 0.38 & 0.34 & 0.56 \\
\hline
\end{tabular}

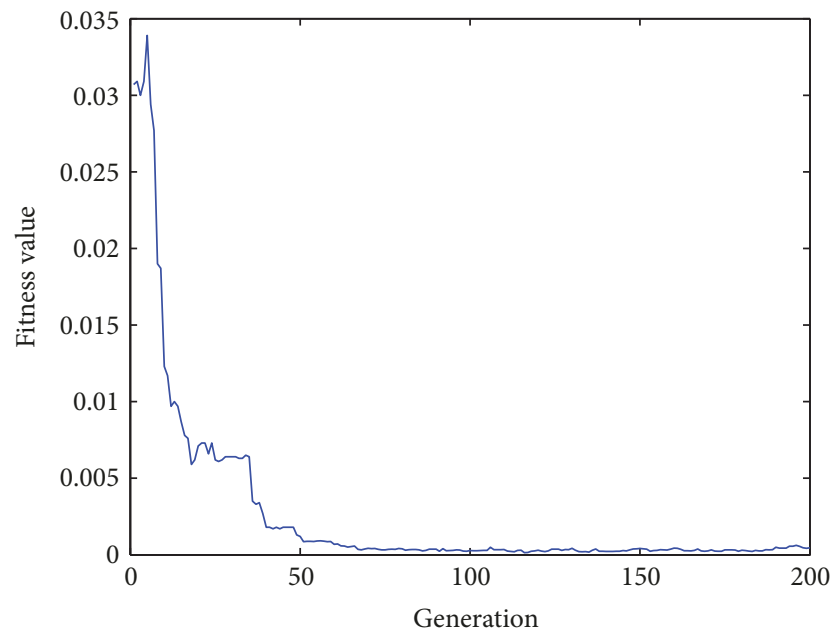

FIGURE 3: The average convergent speed of IHYE algorithm under each state.

after 50 generations, which greatly reduces the running time in practice.
Analyzing the difference return results among the IHYE model, the traditional MV model, and the MCMC method, we can see that the IHYE model has a strong ability to resist risks and gain the biggest long-term benefits. On the one hand, with similar risks, the optimal portfolio of each asset is in direct proportion to the fuzzy expectation of returns seen in Table 2. In each state of the MCMC method, the optimal performance in terms of the expected return leads to higher investment in the next state. In the IHYE model; however, because of the existence of hybrid entropy that measures portfolio risk, the rate of investment in higher risk assets will decrease in spite of the higher expect return. As an example, consider that, in state 2, the proportion of stock 4 increases although the expected return on stock 1 is higher than that on stock 4 . The reason for the IHYE model performing in this situation is that the expectation is just one aspect of choice making: the membership function that calculates the result of hybrid entropy is another aspect of portfolio selection. On the other hand, even with the same GA, the proportion for each stock given by the MV model do not change much: the biggest fluctuation in the IHYE model is seen in state 4, 10.97\%, 


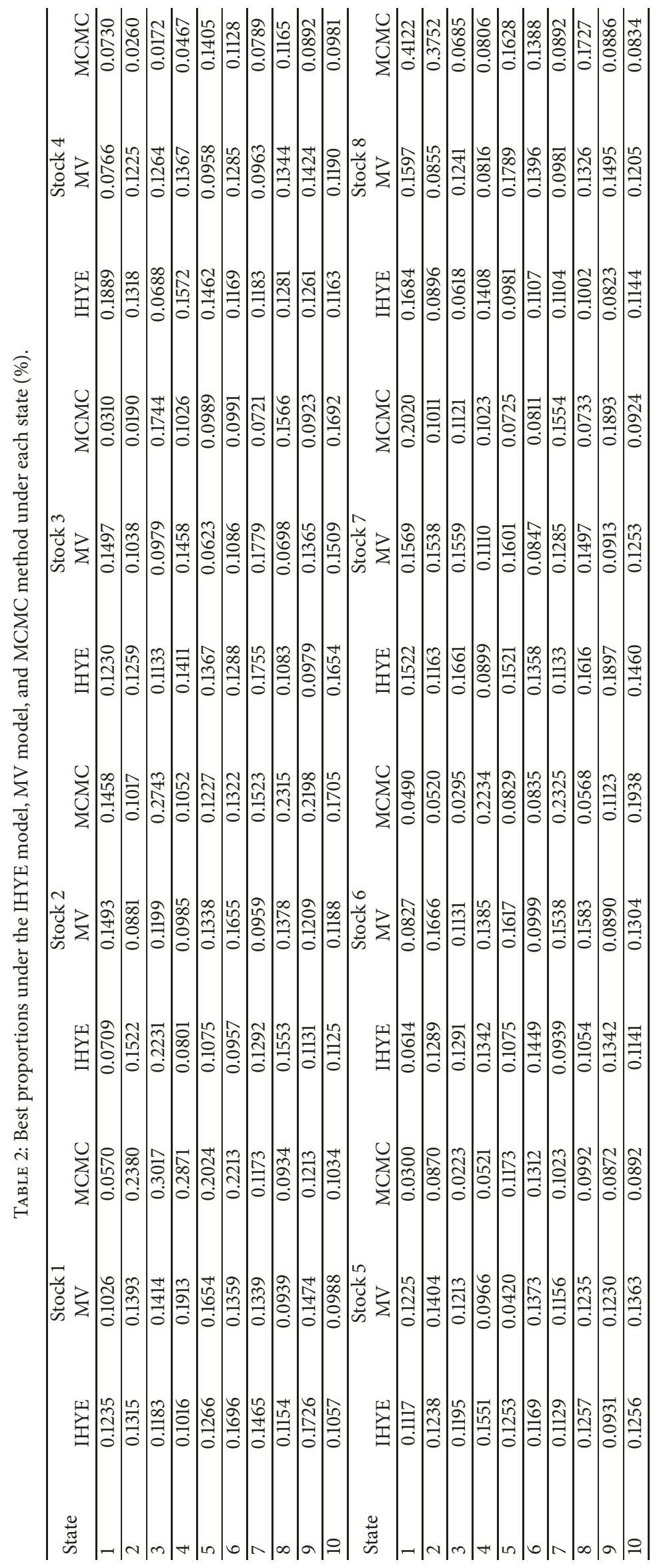


TABLE 3: Longtime portfolio returns on the IHYE and MV models, and the MCMC method.

\begin{tabular}{lccccccccccc}
\hline State & 1 & 2 & 3 & 4 & 5 & 6 & 7 & 8 & 9 & 10 & Sum \\
\hline IHYE & 107.63 & 70.57 & 2.91 & 23.26 & 14.39 & 17.86 & -59.73 & -23.00 & -11.85 & 21.86 & 163.90 \\
\hline MV & 62.65 & 66.30 & -8.90 & 15.24 & -0.45 & 32.15 & -101.58 & -15.5 & -15.96 & 18.23 & 52.18 \\
\hline MCMC & 60.58 & 50.05 & -8.52 & 10.03 & -5.25 & 22.01 & -91.93 & -17.60 & -15.49 & 22.74 & 26.62 \\
\hline
\end{tabular}

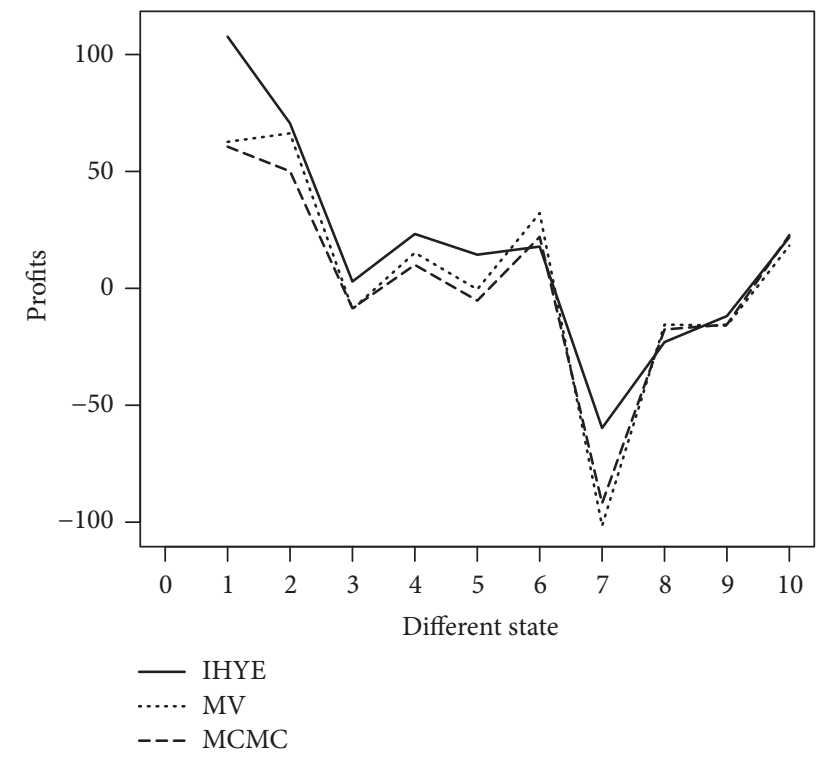

FIgURE 4: Comparing the returns from the 3 models under different states.

which is far lower than the corresponding figures of $22.93 \%$ and $24.04 \%$ for the MV model and the MCMC method.

Based on the return shown in Figure 1, the results for each state and the longtime profits for different methods are given in Table 3 (see also Figure 4). Obviously, the IHYE model has higher longtime profits. There are possibly two reasons contributing to this phenomenon. First, incremental entropy increases returns rapidly. Second, the IHYE model performs best when average returns fall seriously, as shown in Figure 4, which means that hybrid entropy overcomes the shortcomings of using variance and Yager's entropy: it suitably disperses unsystematic risks. Thus, in a longtime trade market, IHYE holds many advantages for investors to earn huge profits at low risks.

Compared with other multiobjective portfolio models, the IHYE model proposed in this paper has the following two advantages, that is, maximizing investors' returns and improving algorithm effectiveness. For investors return, the proposed model ensures the maximization of investors' longterm profits. Compared with the classical MV model and MCMC method from the same period, the empirical results show that the model proposed in this paper performs better in antirisk and profit increasing to guarantee the optimal investment return under each state. Meanwhile, the proposed model assumes that the return rate of securities is a fuzzy random variable where the historical asset return, experts' trading experience, and investors' future expectation are all taken into account. Therefore, it not only considers the fuzzy random uncertainty in the stock market, but also establishes different fuzzy stochastic multiobjective portfolio selection models for different investors to satisfy their different requirements.

For algorithm optimization, Steuer et al. [33] gave a general description to the multiobjective portfolio selection problem. In their research, the effective frontier surface of solutions is obtained by using parametric quadratic programming. However, the requirement of investors in real market is to find a satisfactory portfolio strategy on the effective frontier surface, and traditional methods cannot directly recommend a proper investment ratio for the decision-makers. The algorithm advantage of this paper is to design the compromise genetic algorithm for multiobjective programming problem, providing investors optimal investment strategy among a large number of effective solutions on the efficient frontier surface according to investors preferences. That is, this algorithm reduces the investors difficulty in judging effective solutions. In addition, the proposed algorithm can directly provide a compromise investment strategy in any case, which can effectively handle complex constraints in the model and has a strong advantage in solving multiobjective $(>=3)$ portfolio selection problem.

\section{Conclusion}

In this paper, we firstly construct an incremental-hybridYager's entropy model based on the state-change rule to help select the dynamic portfolio strategy. To obtain longtime profits, feedback effects are used to maximize long-term returns. Fuzzy variables calculated by using Markov chain are built to obtain accurate profit estimates. Furthermore, the proposed model is computed by establishing a compromised GA and obtaining the feasible-investment proportion. Finally, a numerical example is given to show the advantages of this model as compared to the mean-variance model and the MCMC method. In future studies, more complex constraints would be used to avoid correlation ignorance and more closely resemble a real market.

\section{Appendix}

\section{A. Proof of Proposition 7}

We assume that the adjustment policies of portfolios are affine functions about their one-period backwards return deviations, where the coefficients of these functions become the decision variables of the problem. That is to say, the adjustment amount of the portfolio at state $i$ depends on the 
return deviation of the portfolio at state $i-1$. Under this hypothesis, the dynamic feedback adjustment policies can be expressed as the following causal functions:

$$
\begin{aligned}
\Delta \widetilde{\boldsymbol{\beta}}(0) & =\Delta \overline{\boldsymbol{\beta}}(0) \\
\Delta \widetilde{\boldsymbol{\beta}}(i) & =\Delta \overline{\boldsymbol{\beta}}(i)+\Theta(i-1)[\mathbf{R}(i-1)-\widehat{\mathbf{R}}(i-1)] .
\end{aligned}
$$

Then the iterative dynamic equation of the investment proportion of the portfolio at period $i+1$ can be expressed by

$$
\begin{aligned}
\widetilde{\boldsymbol{\beta}}(i+1) & =\widetilde{\boldsymbol{\beta}}(i)+\Delta \widetilde{\boldsymbol{\beta}}(i+1) \\
& =\widetilde{\boldsymbol{\beta}}(i)+\Delta \overline{\boldsymbol{\beta}}(i+1)+\Theta(i)[\mathbf{R}(i)-\widetilde{\mathbf{R}}(i)],
\end{aligned}
$$

where $\mathbf{R}(i)-\widehat{\mathbf{R}}(i)$ is the vector of the fuzzy deviation of the portfolio at period $i$. Then we can obtain the general formula of the portfolio at period $i$ as follows:

$$
\begin{aligned}
\widetilde{\boldsymbol{\beta}}(i) & =\widetilde{\boldsymbol{\beta}}(0) \\
& +\sum_{k=1}^{i}\{\Theta(k-1)[\mathbf{R}(k-1)-\widehat{\mathbf{R}}(k-1)]+\Delta \overline{\boldsymbol{\beta}}(k)\},
\end{aligned}
$$

where $\tilde{\boldsymbol{\beta}}(0)=\boldsymbol{\beta}(0)$ is the investment proportion of the portfolio at the beginning of period 1 .

As asset returns under each state are fuzzy variables, from Definition 1 and the above formulation, we know that

$$
\begin{aligned}
\boldsymbol{\beta}(i) & =\mathrm{E}(\widetilde{\boldsymbol{\beta}}(i-1)+\Delta \widetilde{\boldsymbol{\beta}}(i))=\mathrm{E}(\widetilde{\boldsymbol{\beta}}(i-1) \\
+ & \Theta(i-1)(\mathbf{R}(i-1)-\widehat{\mathbf{R}}(i-1))+\Delta \overline{\boldsymbol{\beta}}(i)) \\
& =\boldsymbol{\beta}(0)+\sum_{k=1}^{i}(\Theta(k-1) \mathrm{E}(\mathbf{R}(k-1)-\widehat{\mathbf{R}}(k-1)) \\
& +\Delta \overline{\boldsymbol{\beta}}(k)) .
\end{aligned}
$$

$$
\begin{aligned}
\mathrm{E}\left(H_{4}\right)= & \mathrm{E}\left\{\sum_{i=1}^{C} G(i) \sum_{h=1}^{m}\left[\beta_{h}(0) R_{h l}(i)+\sum_{k=1}^{i} \sum_{j=1}^{m} \theta_{h j}(k-1) \mathrm{E}\left(R_{h l}(i-1)-\widehat{R}_{h l}(i-1)\right) R_{h l}(i)+\sum_{k=1}^{i} \Delta \bar{\beta}_{h}(k) R_{h l}(i)\right]\right\} \\
= & \sum_{i=0}^{C} G(i)\left\{\sum_{h=1}^{m} \beta_{h}(0) \mathrm{E}\left(R_{h l}(i)\right)+\sum_{h=1}^{m} \sum_{k=1}^{i} \Delta \bar{\beta}_{h}(k) \mathrm{E}\left(R_{h l}(i)\right)\right. \\
& \left.+\sum_{h=1}^{m} \sum_{k=1}^{i} \sum_{j=1}^{m} \theta_{h j}(k-1) \mathrm{E}\left(R_{h l}(i-1)-\widehat{R}_{h l}(i-1)\right) \mathrm{E}\left(R_{h l}(i)\right)\right\},
\end{aligned}
$$

Thus, the investment proportion of risky asset $h$ at period $i$ can be expressed by

$$
\begin{aligned}
& \beta_{h}(i)=\beta_{h}(0) \\
& +\sum_{k=1}^{i}\left(\sum_{j=1}^{m} \theta_{h j}(k-1) \mathrm{E}\left(R_{j}(k-1)-\widehat{R}_{j}(k-1)\right)\right. \\
& \left.+\Delta \bar{\beta}_{h}(k)\right) .
\end{aligned}
$$

The proof is thus complete.

\section{B. Proof of Proposition 8}

In the short term, we have

$$
\begin{aligned}
H_{4} & =\sum_{i=1}^{C}\left[G(i) \ln \left(\sum_{h=1}^{m} \beta_{h}(i)\left(1+R_{h l}(i)\right)\right)\right] \\
& =\sum_{i=1}^{C}\left[G(i) \ln \left(1+\sum_{h=1}^{m} \beta_{h}(i) R_{h l}(i)\right)\right],
\end{aligned}
$$

Further, $\sum_{h=1}^{m} \beta_{h}(i) R_{h l}(i) \longrightarrow 0$, so $\ln \left(1+\sum_{h=1}^{m} \beta_{h}(i) R_{h l}(i)\right) \longrightarrow$ 0 , and based on Taylor's expansion, we have

$$
\begin{aligned}
H_{4} & =\sum_{i=1}^{C}\{G(i) \\
& \left.\cdot\left(\sum_{h=1}^{m} \beta_{h}(i) R_{h l}(i)+o\left(\sum_{h=1}^{m} \beta_{h}(i) R_{h l}(i)\right)\right)\right\},
\end{aligned}
$$

where

$$
\begin{aligned}
& \beta_{h}(i)=\beta_{h}(0) \\
&+ \sum_{k=1}^{i}\left(\sum_{j=1}^{m} \theta_{h j}(k-1) \mathrm{E}\left(R_{j}(k-1)-\widehat{R}_{j}(k-1)\right)\right. \\
&\left.+\Delta \bar{\beta}_{h}(k)\right) .
\end{aligned}
$$

Taking the expectation of (B.2) yields 
Let $K_{1}=\sum_{i=0}^{C} G(i), K_{2}(i)=\sum_{k=1}^{i} \sum_{j=1}^{m} \theta_{h j}(k-1) \mathrm{E}\left(R_{h l}(i-1)-\right.$ $\left.\widehat{R}_{h l}(i-1)\right)$, and $K_{3}(i)=\sum_{k=1}^{i} \Delta \bar{\beta}_{h}(k)$.

Then, from Definition 2 and (16), we have

$$
\begin{aligned}
\mathrm{E}\left[R_{h l}(i)\right]= & \mathrm{E}\left[\mu_{R_{h l}(i)}(x(i))\right] \\
= & \int_{a_{h l}(i)}^{b_{h l}(i)} \frac{x(i)-a_{h l}(i)}{b_{h l}(i)-a_{h l}(i)} \mathrm{d} x(i) \\
& +\int_{b_{h l}(i)}^{b_{h l}(i)} 1 \mathrm{~d} x(i) \\
& +\int_{b_{h l}(i)}^{c_{h l}(i)} \frac{c_{h l}(i)-x(i)}{c_{h l}(i)-b_{h l}(i)} \mathrm{d} x(i) \\
= & \frac{c_{h l}(i)-a_{h l}(i)}{2},
\end{aligned}
$$

Substituting $\mathrm{E}\left[R_{h l}(i)\right]$ into $\mathrm{E}\left(H_{4}\right)$, we can obtain equation (4). The proof is thus complete.

\section{Proof of Proposition 10}

Consider a function $Q(P)$ that measures the distance in $n$ space of a probability distribution $P$ from the vector $[1 / m]$. In some sense this distance can be seen as a measure of certainty associated with the probability distribution. We shall refer to $Q(P)$ as a measure of neg-entropy since the further an element is from $[1 / m]$, the more certain and the lower its entropy.

Yager [31] developed a measure of neg-entropy as follows:

$$
Q\left(\beta_{h}\right)=D\left(P,\left[\frac{1}{m}\right]\right)=\left(\sum_{h=1}^{m}\left|\beta_{h}-\frac{1}{m}\right|^{\mathscr{Z}}\right)^{1 / Z},
$$

It is easy to see the symmetry and continuity of this measure. Furthermore, we note that $Q\left(I_{i}\right)=Q\left(I_{j}\right)=Q\left(I_{h}\right)$ and the distance to any singleton is the same. It can be shown that the maximal value for $Q(P)$ occurs when $P=\left[I_{h}\right]$. In this case, where $P=\left[I_{h}\right]$, we get

$$
\begin{aligned}
Q\left(\left[I_{h}\right]\right) & =\left[\left(\frac{m-1}{m}\right)^{z}+(m-1)\left(\frac{1}{m}\right)^{z}\right]^{1 / z} \\
& =\frac{1}{m}\left[(m-1)^{z}+m-1\right]^{1 / z} \\
& =\frac{m-1}{m}\left[1+\frac{1}{(m-1)^{z-1}}\right]^{1 / z},
\end{aligned}
$$

Yager's entropy consists of the maximum distance $Q\left(\left[I_{h}\right]\right)$ and the normal distance $Q\left(\beta_{h}\right)$. Thus, the value of this entropy is

$$
Y\left(\beta_{h}\right)=Q\left(\left[I_{h}\right]\right)-Q\left(\beta_{h}\right),
$$

Consistent with Section $3, Z=2$ and

$$
\begin{aligned}
Q\left(\left[I_{h}\right]\right) & =\left(1-\frac{1}{m}\right)^{1 / 2}, \\
Q\left(\beta_{h}\right) & =\left(\sum_{h=1}^{m} \beta_{h}^{2}-\frac{1}{m}\right)^{1 / 2}
\end{aligned}
$$

and

$$
Y\left(\beta_{h}\right)=\left(1-\frac{1}{m}\right)^{1 / 2}-\left(\sum_{h=1}^{m} \beta_{h}^{2}-\frac{1}{m}\right)^{1 / 2}
$$

As Yager's entropy is only related to the portfolio proportion, the measure of asset diversity under state changes is given as

$$
Y\left(\beta_{h}(i)\right)=\left(1-\frac{1}{m}\right)^{1 / 2}-\left(\sum_{h=1}^{m} \beta_{h}^{2}-\frac{1}{m}\right)^{1 / 2} .
$$

To characterize the approximate degree of the real proportion to the equal proportion, Yager's entropy should be minimized. The proof is thus complete.

\section{Data Availability}

The data used to support the findings of this study are available from the corresponding author upon request.

\section{Conflicts of Interest}

The authors declare that they have no conflicts of interest.

\section{Acknowledgments}

This work was supported by the National Natural Science Foundation of China (Grants 11571069 and 11501094), the China Scholarship Council (Grant 201706620031), and the Jilin Province Science and Technology Department (Grant 201705200054JH).

\section{References}

[1] H. Markowitz, "Portfolio selection," The Journal of Finance, vol. 7, no. 1, pp. 77-91, 1952.

[2] S. Giove, S. Funari, and C. Nardelli, "An interval portfolio selection problem based on regret function," European Journal of Operational Research, vol. 170, no. 1, pp. 253-264, 2005.

[3] P. Gupta, M. K. Mehlawat, and A. Saxena, "Asset portfolio optimization using fuzzy mathematical programming," Information Sciences, vol. 178, no. 6, pp. 1734-1755, 2008.

[4] W. Zhang, Y. Liu, and W. Xu, "A possibilistic mean-semivariance-entropy model for multi-period portfolio selection with transaction costs," European Journal of Operational Research, vol. 222, no. 2, pp. 341-349, 2012.

[5] F. B. Abdelaziz, B. Aouni, and R. E. Fayedh, "Multi-objective stochastic programming for portfolio selection," European Journal of Operational Research, vol. 177, no. 3, pp. 1811-1823, 2007.

[6] R. A. Abrams and U. S. Karmarkar, "Optimal multiperiod investment-consumption policies," Econometrica, vol. 48, no. 2, pp. 333-353, 1980.

[7] G. C. Calafiore, "Multi-period protfolio optimization with linear control policies," Automatica, vol. 44, no. 10, pp. 2463$2473,2008$.

[8] G. C. Calafiore, "An affine control method for optimal dynamic asset allocation with transaction costs," SIAM Journal on Control and Optimization, vol. 48, no. 4, pp. 2254-2274, 2009. 
[9] J. Li and J. Xu, "Multi-objective portfolio selection model with fuzzy random returns and a compromise approach-based genetic algorithm," Information Sciences, vol. 220, pp. 507-521, 2013.

[10] N. R. Pal and J. C. Brzdek, "Measuring Fuzzy Uncertainty," IEEE Transactions on Fuzzy Systems, vol. 2, no. 2, pp. 107-118, 1994.

[11] R. D. Luce and H. Raiffa, Games and decisions: introduction and critical survey, Dover Books on Mathematics, 1989.

[12] H. Raiffa, Mc-Graw Hill,.

[13] J. G. Carvalho and C. T. Costa, "Identification method for fuzzy forecasting models of time series," Applied Soft Computing, vol. 50, pp. 166-182, 2017.

[14] C. Cheng and J. Yang, "Fuzzy time-series model based on rough set rule induction for forecasting stock price," Neurocomputing, vol. 302, pp. 33-45, 2018.

[15] A. Al-Refaie, G. Bani Domi, and R. Abdullah, "A fuzzy goal programming-regression approach to optimize process performance of multiple responses under uncertainty," International Journal of Management Science and Engineering Management, pp. 1-13, 2018.

[16] L. Ocampo, "A probabilistic fuzzy analytic network process approach (PROFUZANP) in formulating sustainable manufacturing strategy infrastructural decisions under firm size influence," International Journal of Management Science and Engineering Management, pp. 1-17, 2017.

[17] R. Efendi, N. Arbaiy, and M. M. Deris, "A new procedure in stock market forecasting based on fuzzy random autoregression time series model," Information Sciences, vol. 441, pp. 113-132, 2018.

[18] J. Lorkowski, V. Kreinovich, and R. A. Aliev, “Towards decision making under interval, set-valued, fuzzy, and Z-number uncertainty: a fair price approach," in Proceedings of the IEEE International Conference on Fuzzy Systems (FUZZ-IEEE '14), pp. 2244-2253, Beijing, China, July 2014.

[19] W. Rödder, I. R. Gartner, and S. Rudolph, "An entropy-driven expert system shell applied to portfolio selection," Expert Systems with Applications, vol. 37, no. 12, pp. 7509-7520, 2010.

[20] I. Usta and Y. M. Kantar, "Mean-variance-skewness-entropy measures: A multi-objective approach for portfolio selection," Entropy, vol. 13, no. 1, pp. 117-133, 2011.

[21] X. Huang, "Mean-entropy models for fuzzy portfolio selection," IEEE Transactions on Fuzzy Systems, vol. 16, no. 4, pp. 1096-1101, 2008.

[22] J. Xu, X. Zhou, and D. D. Wu, "Portfolio selection using $\lambda$ mean and hybrid entropy," Annals of Operations Research, vol. 185, pp. 213-229, 2011.

[23] P. Jana, T. K. Roy, and S. K. Mazumder, "Multi-objective possibilistic model for portfolio selection with transaction cost," Journal of Computational and Applied Mathematics, vol. 228, no. 1, pp. 188-196, 2009.

[24] R. Zhou, R. Cai, and G. Tong, "Applications of entropy in finance: a review," Entropy. An International and Interdisciplinary Journal of Entropy and Information Studies, vol. 15, no. 11, pp. 4909-4931, 2013.

[25] Y. Fang, K. K. Lai, and S.-Y. Wang, "Portfolio rebalancing model with transaction costs based on fuzzy decision theory," European Journal of Operational Research, vol. 175, no. 2, pp. 879-893, 2006.

[26] J.-R. Yu, W.-Y. Lee, and W. J. P. Chiou, "Diversified portfolios with different entropy measures," Applied Mathematics and Computation, vol. 241, pp. 47-63, 2014.
[27] B. Liu and Y.-K. Liu, "Expected value of fuzzy variable and fuzzy expected value models," IEEE Transactions on Fuzzy Systems, vol. 10, no. 4, pp. 445-450, 2002.

[28] L. M. D. C. Ibáñez and A. G. Muñoz, "A subjective approach for ranking fuzzy numbers," Fuzzy Sets and Systems, vol. 29, no. 2, pp. 145-153, 1989.

[29] J. Ou, "Theory of portfolio and risk based on incremental entropy," The Journal of Risk Finance, vol. 6, no. 1, pp. 31-39, 2005.

[30] X. G. Shang and W. S. Jiang, "Comments on hybrid entropies," Control Theory \& Applications. Kongzhi Lilun yu Yingyong, vol. 16, no. 1, pp. 84-86, 1999.

[31] R. R. Yager, "Measures of entropy and fuzziness related to aggregation operators," Information Sciences, vol. 82, no. 3-4, pp. 147-166, 1995.

[32] J.-R. Yu, W.-J. P. Chiou, W.-Y. Lee, and K.-C. Yu, "Does entropy model with return forecasting enhance portfolio performance?" Computers \& Industrial Engineering, vol. 114, pp. 175-182, 2017.

[33] R. E. Steuer, Y. Qi, and M. Hirschberger, "Multiple objectives in portfolio selection," Journal of financial decision making, vol. 1, no. 1, pp. 11-26, 2005. 


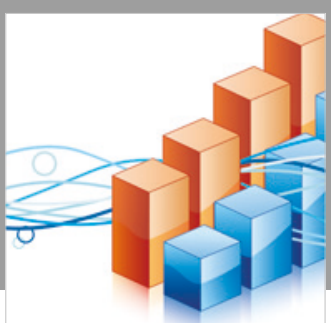

Advances in

Operations Research

\section{-n-m}
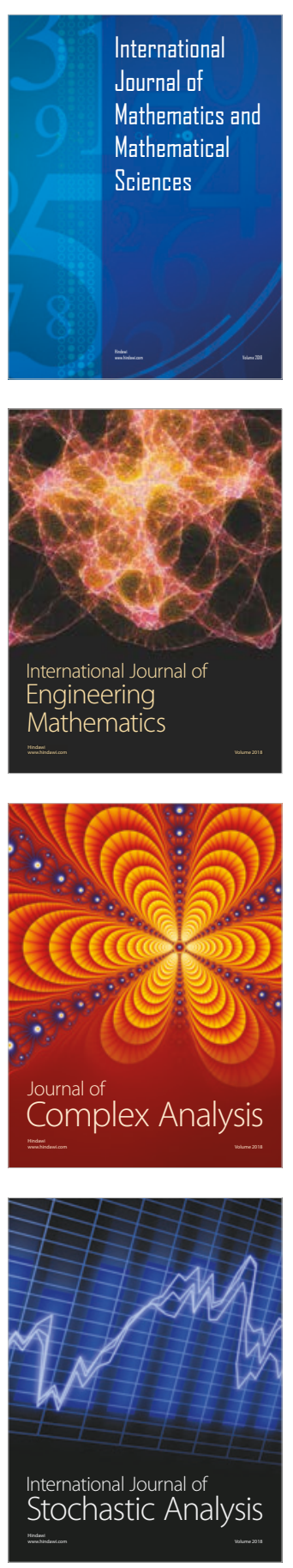
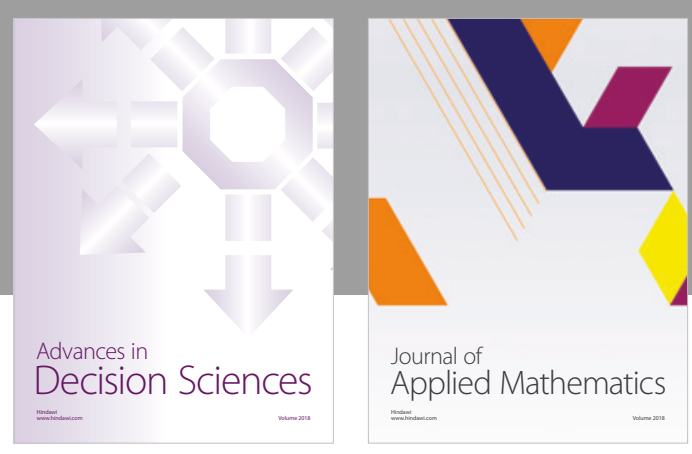

Journal of

Applied Mathematics
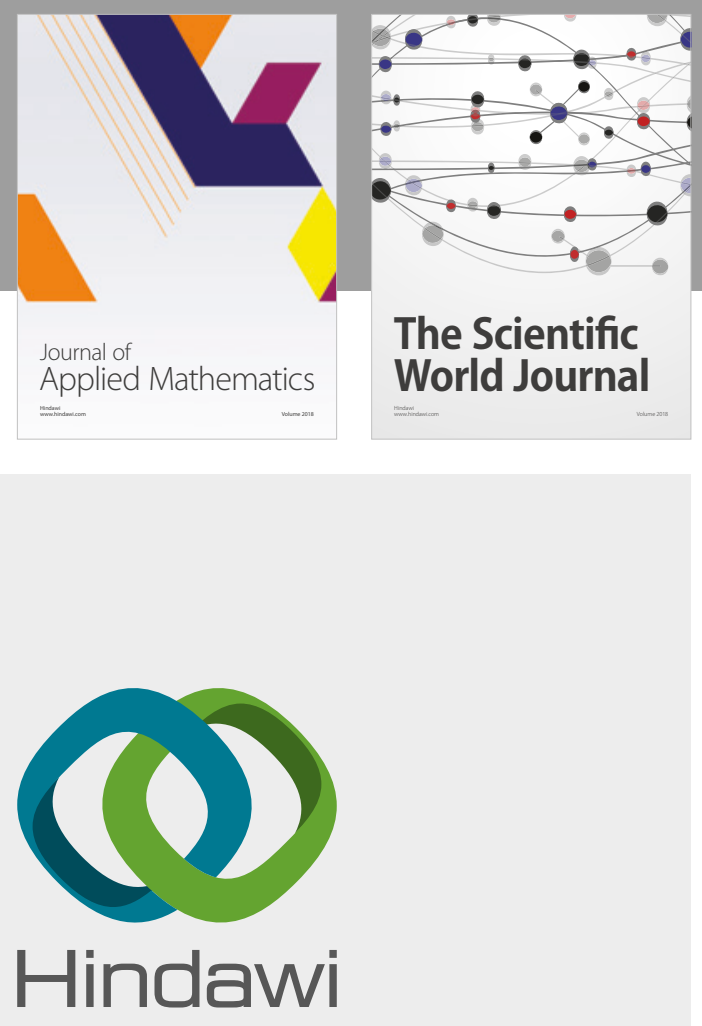

Submit your manuscripts at

www.hindawi.com

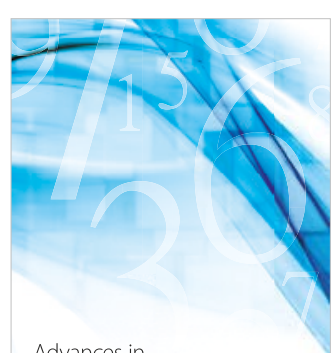

Advances in
Numerical Analysis
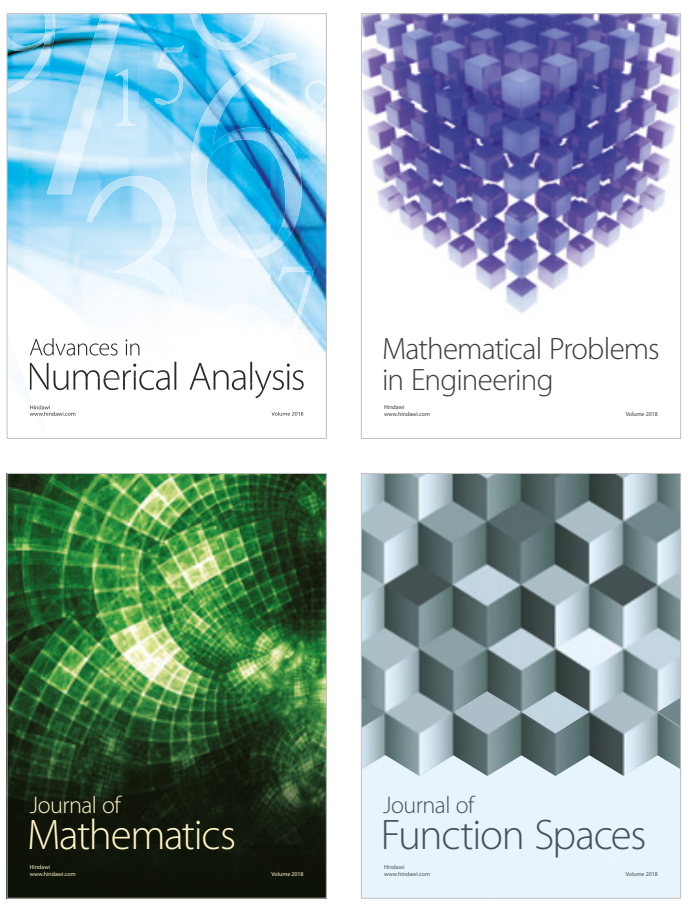

Mathematical Problems in Engineering

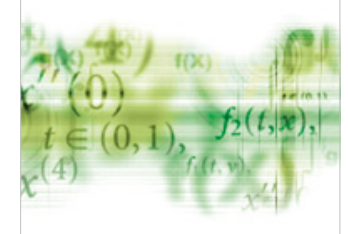

International Journal of

Differential Equations

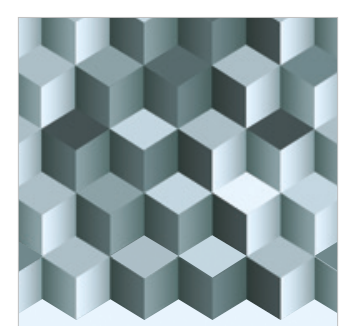

Journal of

Function Spaces

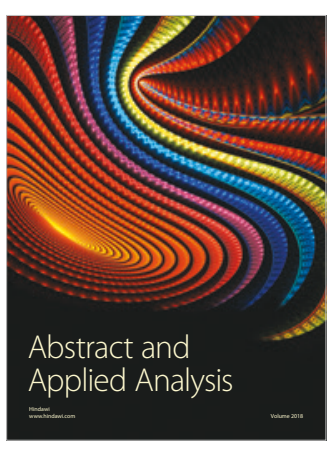

The Scientific

World Journal

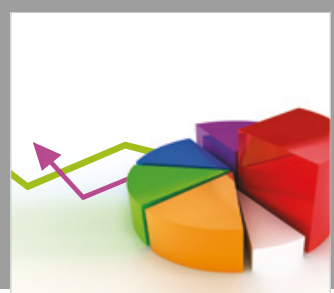

Journal of

Probability and Statistics
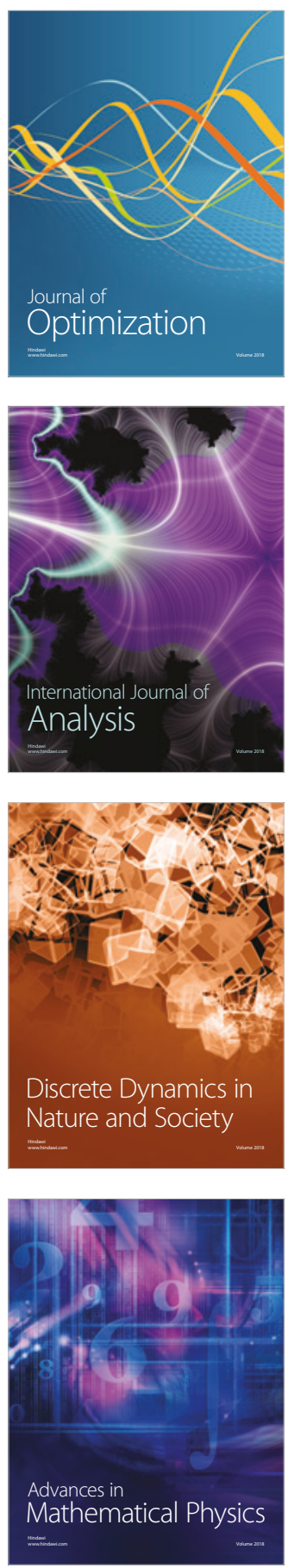